\title{
Engenharia e Design - Aditivo, contra o COVID-19
}

\author{
https://doi.org/10.21814/uminho.ed.24.19
}

\section{António J. Pontes}

\begin{abstract}
António J. Pontes (ORCID: 0000-0002-8964-400X) é Professor Associado no Departamento de Engenharia de Polímeros da Escola de Engenharia da Universidade do Minho. Desenvolve a sua atividade de investigação nas áreas do desenvolvimento do produto e processamento avançado de polímeros. É Investigador Responsável de vários projetos com a indústria e, desde 2012, coordena a parceria entre a Universidade do Minho e a Bosch Brg. É vice-presidente do PIEP associação e CEO do DONE Lab - Manufatura Avançada de Produtos e Ferramentas.
\end{abstract}

\section{Álvaro M. Sampaio}

Álvaro M. Sampaio (ORCID: 0000-0002-8635-1682) é Designer Industrial e Professor Auxiliar na Escola de Arquitetura da Universidade do Minho, com Mestrado em Design, Materiais e Gestão do Produto e Doutoramento em Engenharia de Polímeros. Dedica a sua investigação ao design e desenvolvimento de produto, nas áreas do design centrado no utilizador e na tecnologia de fabrico aditivo. É investigador no grupo Detech do Lab2PT e COO do DONE Lab - Manufatura Avançada de Produtos e Ferramentas. 


\section{INTRODUÇÃO}

À medida que muitos de nós nos adaptamos a novas rotinas e tomamos precauções adicionais para cuidar da saúde e do bem-estar das nossas famílias e colegas, fomos ficando familiarizados com a natureza crescente da crise do coronavírus. "Confinamento", "distanciamento social", "estado de emergência nacional" foram termos que começaram a fazer parte do nosso dia-a-dia pela forma como vieram alterar os nossos hábitos diários. Já passaram quatro meses de uma nova realidade que diminuiu drasticamente a nossa mobilidade e nos colocou num estado de inércia, nunca antes visto (Figura 1).

Há medida que o número de casos aumenta por todo o mundo, as fonteiras foram fechadas, como forma de estancar a propagação da doença e todos os esforços são direcionados para a sobrevivência dos serviços de prestação de saúde, como salvaguarda dos meios de combate à pandemia. Contudo, estas medidas preventivas dizimaram as cadeias de abastecimento de todo o mundo. Os produtos e equipamentos que chegavam habitualmente ao destino final ou intermédio, deixaram de chegar nos prazos previstos e muitos deles num número muito limitado relativamente à procura.

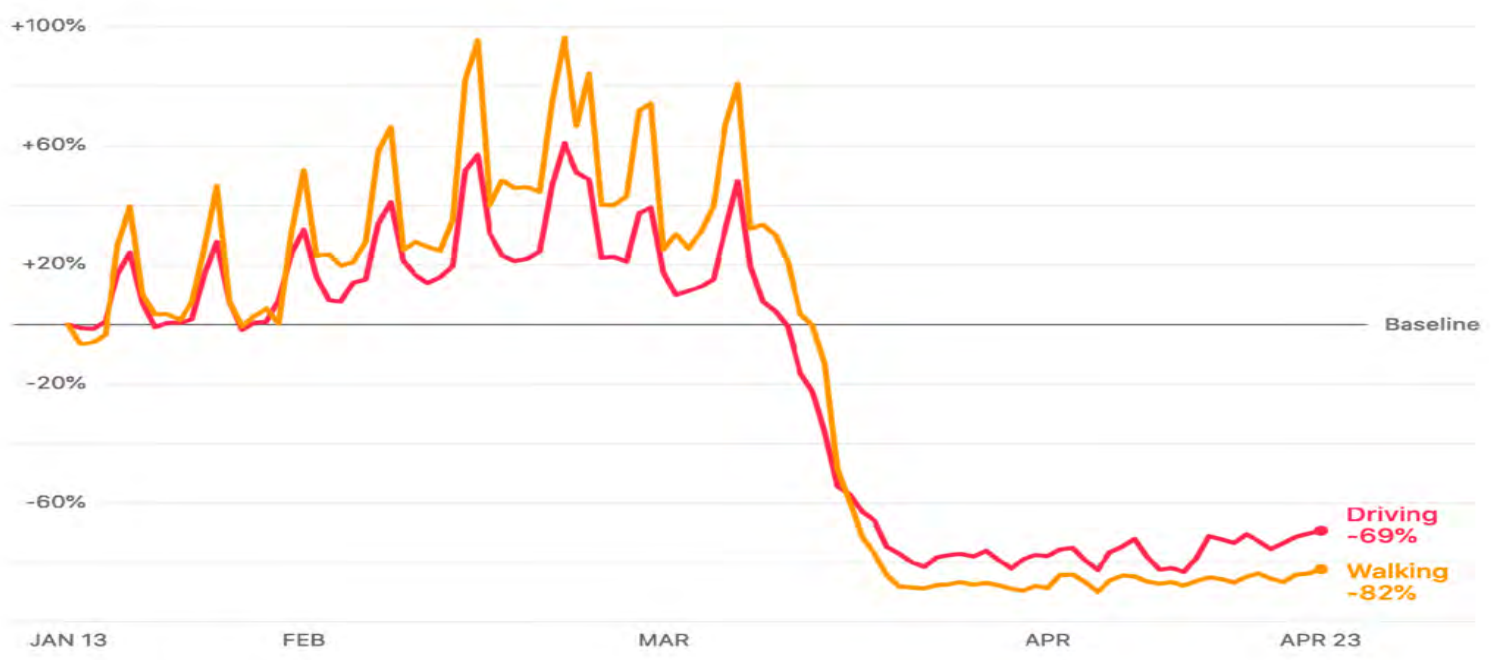

Figura 1. Tendências de mobilidade em Portugal entre 13 de janeiro e 23 de abril de 2020 (Apple, 2020). 
O facto de a China ter sido o foco desta pandemia, e ter estado fechada durante meses, foi também um fator determinante para esta quebra de diferentes cadeias de abastecimento, tanto ao nível de sistemas, produtos, subprodutos, como de matéria-prima.

O aumento generalizado de casos, e subsequentemente de casos graves, sobrecarregou os serviços de saúde amplificando as suas necessidades, e obrigando a pedidos generalizados de dispositivos médicos e equipamentos de proteção individual (EPI) que foram rapidamente consumidos ou que nunca foram equacionados em tão grande número. Neste quadro, rapidamente ficou claro que o convencional canal de resposta a estes pedidos estava destruído. Muitas empresas pararam a produção, especialmente os setores em que a distância entre as pessoas não podia ser garantida. Foi necessário recorrer a processos de fabrico flexíveis e passiveis de produzir produtos em média e larga escala recorrendo a processos tradicionalmente usados na produção de pequenas séries. Exemplo disso foi o uso generalizado de equipamentos de impressão 3D em rede, para produção de pequenos produtos ou acessórios. A produção por Fabrico Aditivo (FA) é um processo de fabrico digital em que não são necessários moldes, cortantes, e outras ferramentas produtivas, em que um único operador pode cuidar de uma série de máquinas numa área que está, também ela, bem controlada. Estas condiçóes fizeram com que o fabrico aditivo se tenha tornado a tecnologia ideal para ajudar a sociedade nestes tempos de pandemia, com produtos individuais e a pedido.

\section{O ADITIVO}

O FA, comummente designado como impressão 3D, permite produzir componentes de elevada complexidade, camada a camada com poucas restriçóes geométricas, quando comparadas com processos tecnológicos de fabrico convencionais. Cada camada sucessiva de material é fundida com a camada anterior pela aplicação de energia térmica, ligantes ou agentes de cura.

Atualmente estão disponíveis uma variedade de tecnologias e equipamentos de FA para diferentes tipos de polímeros, metais, ligas, compósitos, cerâmica e resinas. Estes processos de FA permitem uma criação fácil de geometrias complexas sem ser necessária uma vasta experiência de fabrico e ferramentas moldantes. Isto 
acontece porque a informação digital da modelação sólida é convertida num ficheiro de formato (e.g. tipicamente STL, SLC ou VRML), aceite pelo software proprietário de preparação de produção.

Com os avanços tecnológicos dos processos e materiais, o FA tem vindo a ser adotado como uma tecnologia, não só para produção de protótipos (ie., Rapid Prototyping), mas igualmente na produção de peças funcionais. Entre as vantagens oferecidas pelo FA, podemos destacar: (i) liberdade geométrica das peças sem necessidade de usar outras ferramentas; (ii) redução do tempo de desenvolvimento do produto; (iii) diminuição do custo associado à complexidade da peça ou a sua customização e (iv) libertar o potencial de funcionalização dos componentes.

Neste âmbito, o FA, tem sido há muito anunciado como o prenúncio de uma nova revolução industrial (Anderson, 2014). Com uma máquina pequena e um pouco de paciência, qualquer um seria capaz de imprimir qualquer coisa no conforto do seu lar. Esta tecnologia apresenta alguns benefícios sobre as técnicas tradicionais de fabrico: a prototipagem é mais rápida, a personalização é mais fácil, e as máquinas podem ser configuradas em quase qualquer lugar, o que significa que se pode produzir muito mais perto de onde os produtos precisam de estar. Contudo, ao nível industrial, uma das maiores vantagens do FA é o facto de não serem necessárias ferramentas produtivas (eg., moldes), uma vez que é possível fabricar diretamente os produtos. Este papel das tecnologias de FA está eminentemente ligado a uma aplicação que durante vários anos foi utilizada na indústria, e que se trata da produção de pequenas séries ou o chamado bridge development, que é um processo ágil de comercializar um produto antes de produzir um molde para a produção de grandes séries. Neste âmbito, o FA é capaz de desempenhar um papel muito importante na ajuda ao apoio às cadeias de abastecimento afetadas por limitações de produção e consequentemente de importações, nomeadamente o FA pode ter um efeito benéfico imediato quando essas cadeias estão totalmente interrompidas. Esse foi, felizmente, o caso quando a pandemia COVID-19 interrompeu seriamente as cadeias de abastecimento de diferentes indústrias em todo o mundo, principalmente a relacionada a produtos e dispositivos médicos. 


\section{O ADITIVO NA COVID}

Quando a propagação de COVID-19 interrompeu as cadeias de abastecimento em todo o mundo, os países procuraram comprar rapidamente e armazenar dispositivos médicos - máscaras faciais, viseiras, zaragatoas, ventiladores, etc. - mas encontraram poucos fornecedores para os abastecer. Em resposta, várias redes foram rapidamente criadas, através da agregação de pessoas individuais que, com o recurso a impressoras 3D (equipamentos de FA) começaram a tentar suprir as necessidades da forma que podiam. A grande generalidade destas iniciativas estava centrada na produção de equipamentos de proteção individual (EPI), tanto para os prestadores de cuidados de saúde como para o público em geral. Estas iniciativas atingiram uma escala global, e rapidamente ficou claro que o FA era importante no combate à pandemia. À medida que governos e os profissionais de saúde pediam aos fabricantes o fornecimento de equipamentos e dispositivos, como ventiladores e equipamento de proteção para ajudar a tratar os pacientes, o FA respondeu ao apelo através da sua facilidade de produção e da sua rede de fornecedores. Tendo sido quase impossível acompanhar o número de iniciativas criadas para ajudar no combate à COVID-19.

Algumas destas iniciativas nasceram pelo dinamismo individual através de redes criadas de movimentos do tipo Makers, que a um nível inicial conseguiram mobilizar muitas pessoas, outras foram altamente organizadas envolvendo parcerias entre países ou diferentes regiões, através de unidades industriais de FA, centradas na produção não só de EPI, mas também em dispositivos médicos. A capacidade de conceber, produzir, testar e iterar peças em questão de horas faz com que o FA se tenha evidenciado como a tecnologia indicada no fabrico de produtos e acessórios para o combate à pandemia da COVID-19. À medida que o clima de incerteza e perturbação foi aumentando, o benefício do FA foi claramente evidenciado, com o desafio de colmatar a deterioração das cadeias de abastecimento. Sendo a velocidade de resposta uma das principais prioridades dos hospitais à espera de abastecimentos, o FA permitiu uma produçãao mais rápida de dispositivos médicos e de proteção individual. Além disso, estes produtos podiam ser fabricados localmente, mais perto do ponto onde seriam utilizados, reduzindo a distância entre o fabricante, o pessoal médico 
e os pacientes. $\mathrm{O}$ facto do FA ser um fabrico digital originou a partilha de modelos virtuais que foram sucessivamente adaptados e otimizados por todo o mundo.

As tecnologias de FA têm também as suas próprias características e especificidades que devem ser consideradas durante a fase de conceção para garantir a qualidade das peças fabricadas. Como tal, a modelos desenvolvidos por curiosos da tecnologia são, rapidamente, associados e disseminados modelos desenvolvidos por designers e engenheiros mais experientes. Estes modelos apresentam melhores características ao nível de eficiência de produção (focado numa tecnologia específica) bem como na qualidade das peças fabricadas. Contudo a partilha de modelos entre curiosos e experts desenvolveu uma rede online sem precedentes. Desenhos, modelos virtuais, materiais, parâmetros e condiçóes de processamento são distribuídos e organizados mediante tecnologias de FA de baixo custo e de produção industrial. A partilha de informação entre indivíduos e entidades que não se conheciam antes, é extraordinária. Os modelos virtuais, os produtos produzidos e os testes realizados para a sua validação são partilhados praticamente sem barreiras. Ou seja, há um crescendo da democratização do conhecimento. 
Tabela 1. Lista de algumas iniciativas de FA criadas para ajudar no combate à COVID-19.

\begin{tabular}{|c|c|c|c|c|}
\hline Local & Entidade & Parceiros & Produtos & Referência \\
\hline \multirow[t]{2}{*}{ EUA } & \multirow[t]{2}{*}{ Carbon } & \multirow[t]{2}{*}{ Universidade de Stanford } & Zaragatoas & \multirow[t]{2}{*}{ Carbon3d, 2020} \\
\hline & & & Viseiras & \\
\hline \multirow[t]{2}{*}{ EUA } & \multirow{2}{*}{$\begin{array}{l}\text { Ford Motor Com- } \\
\text { pany }\end{array}$} & \multirow[t]{2}{*}{ Elix polymers } & Ventiladores & \multirow[t]{2}{*}{ Ford, 2020} \\
\hline & & & Máscaras & \\
\hline UK & 3DPRINTUK & Arts University Bournemouth & Viseiras & $\begin{array}{l}\text { 3DPrint-UK, } \\
2020\end{array}$ \\
\hline Alemanha & MakersVsVirus & 7000 produtores voluntários & EPI & $\begin{array}{l}\text { MakersVsVirus, } \\
2020\end{array}$ \\
\hline China & Winsun & Universidade de Shanghai & $\begin{array}{l}\text { Casas de isola- } \\
\text { mento }\end{array}$ & $\begin{array}{l}\text { Winsun3D, } \\
2020\end{array}$ \\
\hline \multirow[t]{5}{*}{ Espanha } & \multirow[t]{5}{*}{ Card3D } & EIT Health & \multirow[t]{5}{*}{ Máscaras } & \multirow[t]{5}{*}{ EitHealth, 2020} \\
\hline & & Hospital Sant Joan De Déu & & \\
\hline & & Universidade de Barcelona & & \\
\hline & & Fundación CIM-UPC & & \\
\hline & & BCN3D & & \\
\hline \multirow[t]{6}{*}{ Espanha } & \multirow{6}{*}{$\begin{array}{l}\text { Leitat Technology } \\
\text { Center }\end{array}$} & $\mathrm{HP}$ & \multirow[t]{6}{*}{ Ventilador } & \multirow[t]{6}{*}{ Leitat, 2020} \\
\hline & & Seat & & \\
\hline & & Navantia & & \\
\hline & & Airbus & & \\
\hline & & Catsalut & & \\
\hline & & $\begin{array}{l}\text { Hospital Parc Taulí de } \\
\text { Sabadell }\end{array}$ & & \\
\hline \multirow[t]{6}{*}{ Alemanha } & \multirow{6}{*}{$\begin{array}{l}\text { Mobility goes } \\
\text { additive }\end{array}$} & Volkswagen Group & \multirow[t]{6}{*}{ EPI } & \multirow{6}{*}{$\begin{array}{l}\text { Mobility Goes } \\
\text { Additive, } 2020\end{array}$} \\
\hline & & Airbus & & \\
\hline & & Fraunhofer IPT & & \\
\hline & & MotionLab Berlin & & \\
\hline & & Sauter Engineering + Design & & \\
\hline & & +49 & & \\
\hline \multirow[t]{3}{*}{ França } & \multirow[t]{3}{*}{ BONE3D } & Universidade de Paris & \multirow[t]{3}{*}{ Máscaras } & \multirow[t]{3}{*}{ Bone3D, 2020} \\
\hline & & Stratasys & & \\
\hline & & $\begin{array}{l}\text { Assistance publique - Hopi- } \\
\text { taux de Paris }\end{array}$ & & \\
\hline \multirow[t]{2}{*}{ Luxemburgo } & \multirow[t]{2}{*}{ 3D Print } & Universidade do Luxemburgo & \multirow[t]{2}{*}{ EPI } & \multirow[t]{2}{*}{ 3D Print, 2020} \\
\hline & & Goodyear & & \\
\hline
\end{tabular}




\section{O NOSSO ADITIVO}

O FA apresenta algumas vantagens sobre os métodos tradicionais de desenvolvimento de produto e de produção, como:

1) colmatar necessidades críticas imediatas, globais, regionais ou individuais, quer sejam elas EPI, peças de substituição, protótipos, mini, pequenas e médias séries. Neste âmbito, o DONE Lab - Laboratório de Manufctura Avançada de Produtos e Ferramentas, sediado na Universidade do Minho, em Azurém, recebeu, logo no início dos primeiros registos de COVID-19 em Portugal, várias solicitaçôes para produzir EPI para os prestadores de cuidados de saúde, tendo iniciado a produção de viseiras por FA. O DONE Lab dispóe de um conjunto de equipamentos de fabrico digital (aditivo e subtrativo) capaz de produzir produtos e ferramentas apenas recorrendo a informação digital do produto. Assim, aproveitou-se a sua capacidade de produção industrial para se desenvolver e produzir viseiras, especificamente na tecnologia SLS. Esta tecnologia de Power Bed Fusion de polímeros permite, com recurso a um laser e utilizando um material polimérico, a Poliamida 12 em pó, produzir em média escala produtos e componentes sem necessidade de ferramentas moldantes e suportes e, consequentemente, sem necessidade de se realizar tarefas de pós-processamento e acabamento. $\mathrm{O}$ tempo de resposta foi muito curto e rapidamente foram distribuídas cerca de 2000 viseiras (Figura 2).

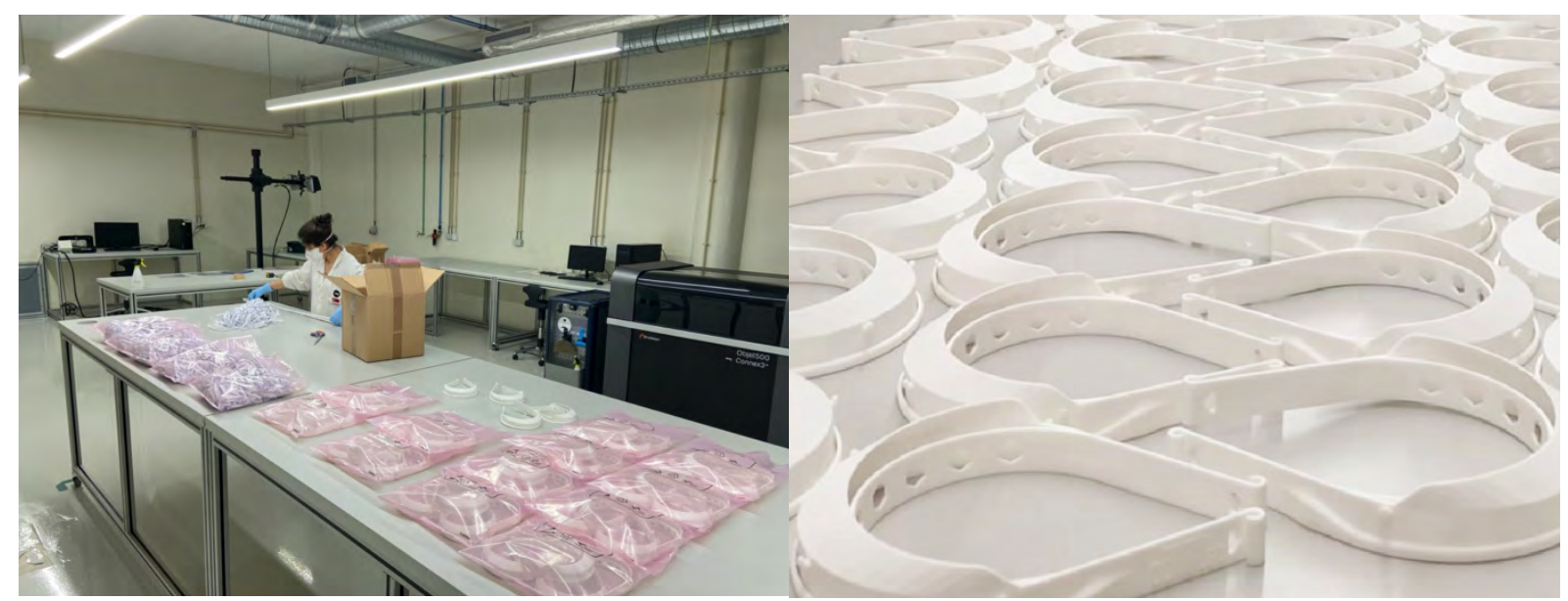

It is important to collaborate closely in these challenging times and contribute to support the people who fight against the COVID-19 pandemic. Everyone can contribute and here we are working to help to keep healthcare workers safe. We are producing face shields, by means of additive manufacturing, which are being donated to "Centro Social de Pedome", to "ACeS do Ave - Famalicão", to "Centro Hospitalar do Médio Ave" in Santo Tirso, and to "Hospital de Braga" (EPE). In these unprecedented times we join forces to help and contribute to the wellbeing of our society.

Figura 2. Produção de uma média-série de viseiras - Publicação do linkedIn, 31 de março de 2020 (DONE Lab, 2020a). 
Isto foi possível porque o FA é uma tecnologia que tem capacidade de se ajustar rapidamente a ambientes em mudança e responder a imprevistos de emergência de uma forma rápida, personalizável, com retorno e satisfação das necessidades quase imediatas (Figura 3).

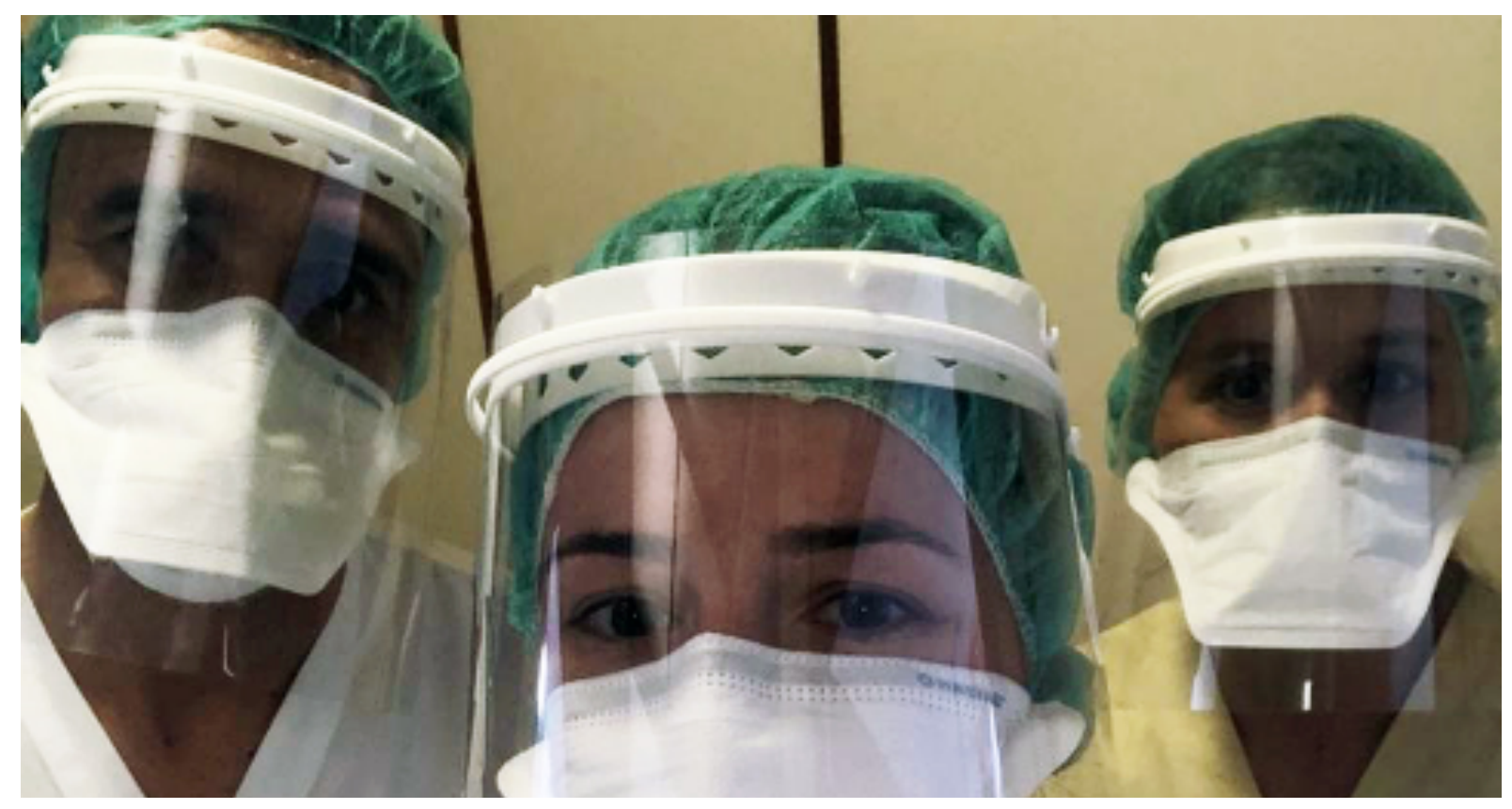

Upon receiving this photo from the "Centro Hospital do Médio Ave" in Santo Tirso (which left us grateful), we feel that we are on the right track. We would like to express our recognition and our deepest gratitude to healthcare workers on front line of COVID-19 fight. We are proud to do our part to support them. We will continue to do our best.

Figura 3. Profissionais de saúde do Centro Hospitalar do Médio Ave - Santo Tirso - Publicação do LinkedIn, 8 de abril de 2020 (DONE Lab, 2020b).

2) possibilitar a adaptação de equipamentos, produtos e sistemas já existentes. Através da informação de base digital e da produção à medida, é possível reutilizar, otimizar e modificar de uma forma simples e quase sem restrições de design qualquer produto ou equipamento. Foi esta capacidade de adaptação que foi implementada no desenvolvimento e produção de componentes para a otimização de máscaras de mergulho (Snorkeling masks) para apoio a ventiladores. Projeto que foi inicialmente desenvolvido pela Isinova em Itália e que o DONE Lab juntamente com o DTx otimizou em colaboração com o Dr. José Miguel Pego do Hospital de Braga, EPE (Figura 4). 
Este projeto surge da constatação do grau de contagiosidade do vírus COVID-19, que é muito elevado, que se transmite facilmente por partículas aerossolizadas da via aérea e que até $10 \%$ dos casos induz um quadro de SARS e/ou pneumonia severa. A agressividade do vírus expõe os profissionais de saúde, em particular, pelo que são recomendadas medidas de isolamento biológico de grau elevado (FP2 para observação dos doentes e FP3 para procedimentos invasivos).

O risco de contágio é maior durante a manipulação da via aérea (e.g., intubação endotraqueal) por exposição aos aerossóis gerados na via aérea. Por outro lado, a evolução natural da doença é benigna e autolimitada, mas em grupos de risco (e.g., idosos, hipertensos, obesos) a evolução é para um SARS com progressão muito rápida para necessidade de ventilação não-invasiva (VNI) e invasiva com internamento em Unidades de Cuidados Intensivos.

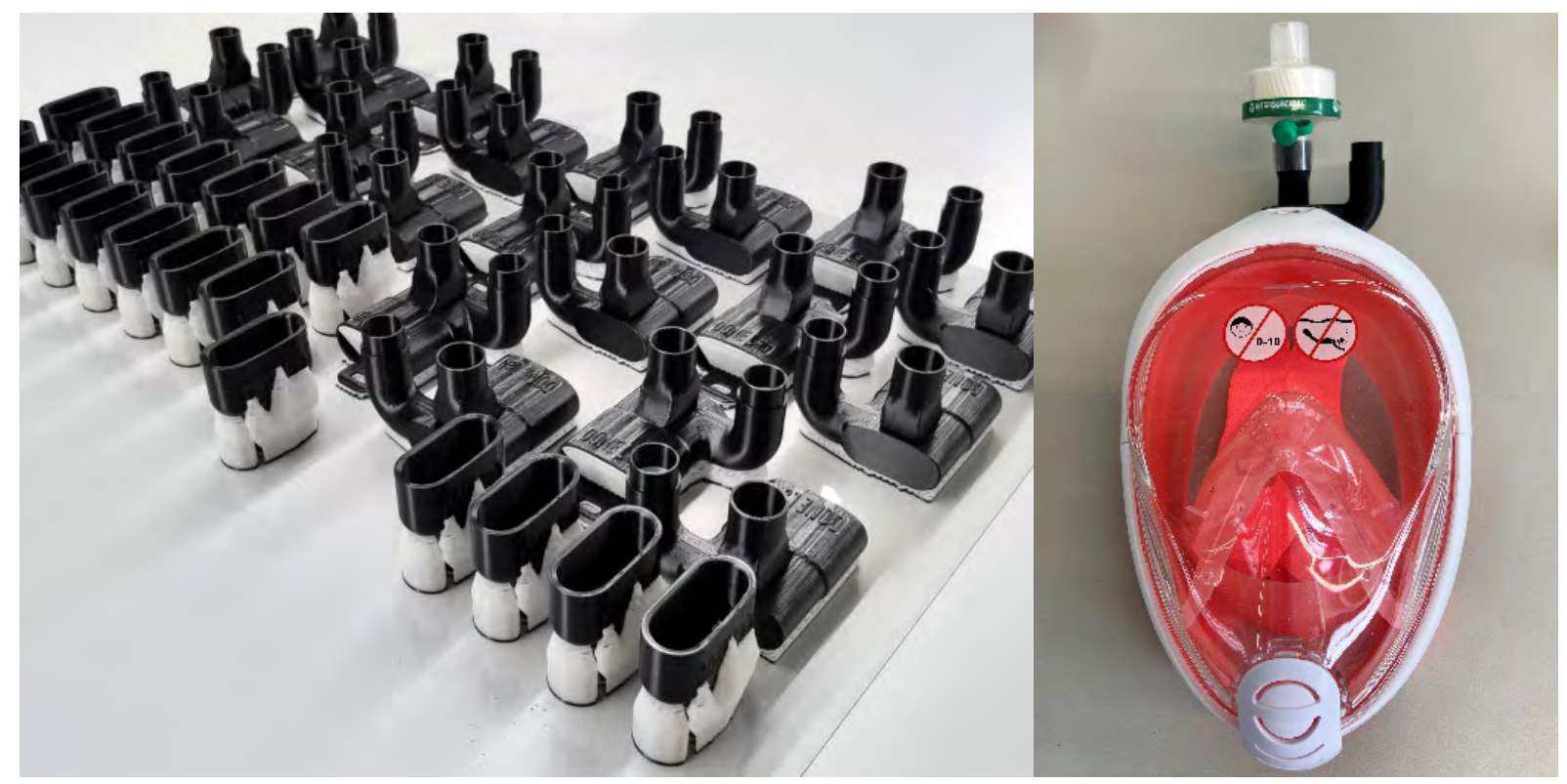

DONE Lab and DTX Colab join forces to help the pandemic fight. We are optimizing, developing and producing new components for emergency ventilator masks, realized by adapting a snorkeling mask already available on the market. These new components are being delivered to Braga Hospital (EPE). The development was based on the concepts of Isinnova.

Figura 4. Adaptação de máscaras de mergulho para apoio a ventiladores - Publicação do LinkedIn, 1 de abril de 2020 (DONE Lab, 2020c). 
Devido a não haver uma imunização de grupo, um período de incubamento prolongado, grau de contagiosidade elevados e um elevado número de infetados assintomáticos, a doença tem uma progressão exponencial que potencialmente leva à exaustão dos recursos dos cuidados de saúde, nomeadamente, EPI e máscaras de VNI.

Existem comercialmente disponíveis, e em stock imediato, máscaras de snorkeling, de proteção integral da face, que poderão servir as funções de EPI ou de VNI com a utilização de adaptadores produzidos por FA, e que façam a interface entre fontes de ar/oxigénio e filtros microbiológicos HEPA. O uso destas máscaras poderá trazer uma resposta imediata à necessidade crescente quer de EPI como de máscaras de VNI, ajudando a melhorar a resposta dos sistemas de saúde à pandemia.

3) possibilidade de sustentar o desenvolvimento e produção de produtos críticos em diferentes escalas e circunstâncias. Desde o colmatar necessidades de pequenas comunidades, ao desenvolvimento em maior escala ultrapassando cadeias de abastecimento interrompidas ou inexistentes, até à ajuda à prevenção de problemas específicos e ou regionais, terminando no suporte à produção em grande escala, quer através da produção de ferramentas rápidas, quer através do bridge development já evidenciado. Foi esta a sustentação que foi evidenciada ao longo do projeto das viseiras que o DONE Lab, em conjunto com uma equipa do Departamento de Engenharia Têxtil (DET) e do Departamento de Engenharia de Polímeros (DEP) da EEUM, em colaboração com a Escola de Arquitetura da Universidade do Minho, encetou uma cooperação com várias empresas. A empresa Ernesto São Simão, após ter realizado estudos e testes prévios realizados com viseiras produzidas por FA, está a produzir um modelo de estrutura para as viseiras por moldação por injeção, tecnologia capaz de produzir as estruturas em poucos segundos e assim responder de forma rápida à procura crescente destes EPI (Figura 5). 


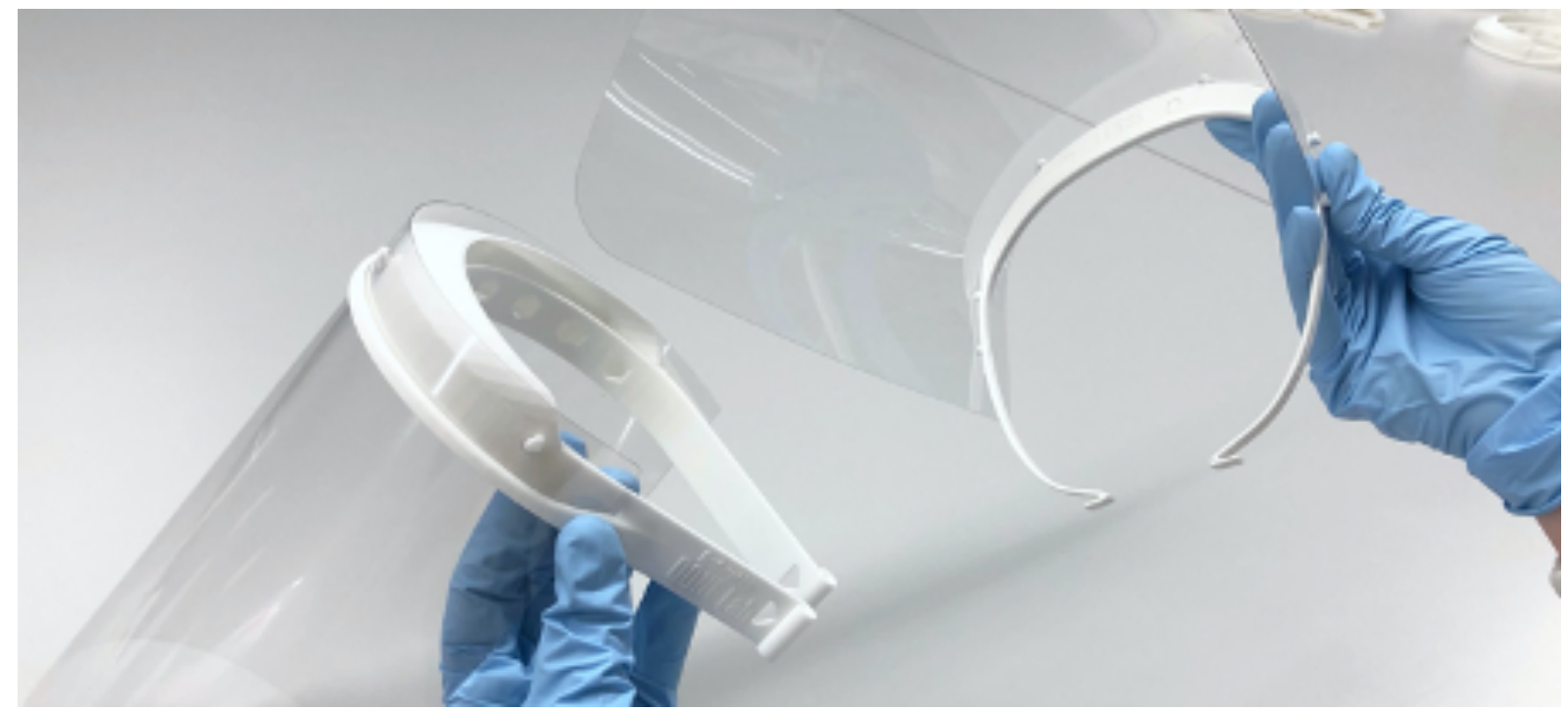

In particularly difficult times we are all encouraged to act responsibly and in collective conscience. Until now over 20000 face shields were delivered to several institutions - IPSS, city councils and companies - in various parts of the country, in a collaboration between the DONE Lab - Advanced Manufacturing of Products and Tools, the University of Minho, the PIEP - Innovation in Polymer Engineering, the Fly London and the Ernesto São Simão, with the support of many other entities. We are proud to be able to contribute with personal protective equipment to everyone's well-being.

Figura 5. Viseiras por FA e por injeção por moldação - Publicação do LinkedIn, 18 de maio de 2020 (DONE Lab, 2020d).

\section{CONCLUSÕES}

As implicações da COVID-19 ainda estão por ser equacionadas na sua totalidade. $\mathrm{O}$ facto do mundo ainda estar a lidar com esta pandemia a isso obriga. $\mathrm{O}$ mesmo se aplica às implicações no FA como indústria, muito porque ainda estamos longe de entender claramente qual será o impacto a curto, médio e longo prazo da pandemia nas cadeias de fornecimento global. Contudo, estes últimos meses vieram tornar claro que com conhecimentos sólidos em Engenharia e saber fazer, associados a um Design inovador, em conjunto com a capacidade, disponibilidade e flexibilidade das tecnologias de FA, é possível produzir produtos e componentes rapidamente (e apenas com informação digital) e assim mitigar a sua escassez em determinados setores de atividade, sobretudo quando situaçóes de emergência interrompem a cadeia de fornecimento, como foi o caso da pandemia COVID-19. 


\section{REFERÊNCIAS BIBLIOGRÁFICAS}

3D Print (2020). Let's Fight Corona Luxembourg. Disponível em: https://www.facebook. com/3dprintletsfightcorona/.

3DPrint-UK (2020). The worlds most efficient 3D printable face shield. Disponível: https://www.3dprint-uk. co.uk/worlds-most-efficient-sls-face-shield-COVID19/.

Anderson, C. (2014). Makers: The new Industrial Revolution. Crown Business, Nova York.

Apple (2020). Mobility trends report. Disponível em: https://www.apple.com/COVID19/mobility.

Consultado em 23 de abril de 2020.

Bone3D (2020). BONE 3D operates the APHP 3DCOVID platform. Disponível em: https://bone3d.com/en/ actualite/bone-3d-operates-the-aphp-3dCOVID-platform/.

Carbon3D (2020). Carbon COVID-19 Response. Disponível em: https://www.carbon3d.com/COVID19/.

DONE Lab (2020a). Produção de uma média-série de viseiras. Publicação do LinkedIn. Disponível em: https://www.linkedin.com/feed/update/urn:li:activity:6650684584323272705.

DONE Lab (2020b). Profissionais de saúde do Centro Hospitalar do Médio Ave - Santo Tirso. Publicação do LinkedIn. Disponível em: https://www.linkedin.com/posts/done-lab digitalmanufacturingadditivemanufacturing-activity-6653624672707452929-mnA1.

DONE Lab (2020c). Adaptação de máscaras de mergulho para apoio a ventiladores. Publicação do LinkedIn. Disponível em: https://www.linkedin.com/posts/done-lab_digitalmanufacturing-additivemanufacturingactivity-6651040171607244801-ZYP3.

DONE Lab (2020d). Viseiras por FA e por injeção por moldação. Publicação do LinkedIn. Disponível em: https://www.linkedin.com/posts/done-lab_bosch-intraplas-joracarsil-activity-6668101582447489024-A0fr.

EitHealth (2020). COVID-19 Rapid Response Innovation project. Disponível em: https://eithealth.eu/ COVID-19-rapid-response/car3d/.

Ford (2020). Doing our part. For us all. Disponível em: https://corporate.ford.com/articles/products/fordcommunity-responds-to-pandemic-on-local-and-national-scale.html.

Leitat (2020). The first medically validated and industrially producible 3D emergency ventilator. Disponível em: https://COVID-leitat.org/en/ventilator/.

MakersVsVirus (2020). Makers vs Virus. Disponível: https://www.makervsvirus.org/.

Mobility Goes Additive (2020). 3D Printing fights Corona. Disponível em: https://mobilitygoesadditive.org/.

Winsun3D (2020). Winsun Makes Global Donation of 3D Printed Isolation Houses. Disponível em: http:// www.winsun3d.com/En/News/news inner/id/547. 